\title{
Tunable Bandwidth of Negative Permittivity from Graphene- Silicon Carbide Ceramics
}

\author{
Zhihao Sun, ${ }^{1}$ Xiaoshuai Huang, ${ }^{1}$ Aiqing Xia, ${ }^{2}$ Zhaoqian Yan $^{1}$ and Lei Qian ${ }^{1, *}$
}

\begin{abstract}
With tunable bandwidth of negative permittivity, graphene-silicon carbide (GR-SiC) ceramics were sintered by spark plasma sintering process. Compositions and structure were investigated by field emission scanning electron microscopy and Raman spectroscopy. With its content increasing, GR interconnected into "GR clusters" with larger lateral dimensions, which tended to be connected into conductive pathways instead of agglomeration under the action of ball milling. Permittivity, alternating current conductivity ( $\mathrm{\sigma ac}$ ), dielectric loss and imaginary reactance were analyzed in detail. When the GR content exceeded $6.30 \mathrm{wt} \%$, a significant electrical percolation phenomenon occurred, and the conductive mechanism changed from electronic jump conduction to a metal-like conduction. Fano-like resonance, appertained to permittivity, exhilaratingly transformed into the Lorentz model. Owing to the mutual dielectric screening between $\mathrm{SiC}$ and $\mathrm{GR}$, the bandwidth of negative permittivity became wider and can be tuned via the GR content.
\end{abstract}

Keywords: Graphene; Negative permittivity; Bandwidth; $\mathrm{SiC}$ ceramics.

Received: 21 August 2021; Accepted: 3 November 2021.

Article type: Communication.

\section{Introduction}

As a novel material, metamaterial with negative permittivity or/and permeability has received extensive attention, which exhibits potential applications in many fields such as electromagnetics, optics and electronics. ${ }^{[1-3]}$ Different from traditional metamaterials fabricated by artificial periodic structures, meta-composites increasingly cause interests of researchers. Meta-composites usually are prepared by common combination of two or more components, and they have many characteristics including rich materials, easily controllable microstructures, simple preparation process, etc. Many meta-composites and corresponding mechanisms have been reported and studied in recent years. It was found that compounding conductive components with insulating matrix can easily produce meta-composites. Conductive materials including graphene, carbon nanotubes, amorphous carbon and metals have been combined with ceramics or resins to prepare meta-composites with negative permittivity. For example, our

\footnotetext{
1 Key Laboratory for Liquid-Solid Structural Evolution and Processing of Materials (Ministry of Education), Shandong University, 17923 Jingshi Road, Jinan 250061, China.

2 College of Chemistry and Chemical Engineering, Xingtai University, Xingtai 054001, China.

*Email: qleric@sdu.edu.cn (L. Qian)
}

group reported reduced graphene oxide (rGO)-alumina ceramics by spark plasma sintering. ${ }^{[4]}$ It was found that negative dielectric performance was tuned by the sintering conditions and the dispersed state of rGO. Cheng et al..$^{[5]}$ prepared the polypyrrole-yttrium iron garnet (YIG) composites, and it was found that the plasma resonance frequency of negative permittivity shifted to the lower frequency with the increasing contents of YIG.

In the previous literature, insulating matrix is often used to construct meta-composites, but a semiconductor as the matrix has rarely been studied and investigated. Silicon carbide ( $\mathrm{SiC})$ is considered as an important wide-bandgap semiconductor material. In recent years, it has a huge potential application prospect in the field of high temperature anti-electromagnetic interference. SiC ceramic exhibits high thermal conductivity, high temperature resistance and thermal shock resistance, and most importantly, it can behave a natural resonance phenomenon at a specific frequency with large loss angle tangent. ${ }^{[6-9]}$ However, the bandwidth of negative permittivity from the natural resonance is narrow, which limits its application. Graphene, as a good conductive filler, can change the interface structure and effectively control the dielectric parameters. Therefore, the realization of the perfect combination of $\mathrm{GR}$ and $\mathrm{SiC}$ is expected to expand the negative dielectric bandwidth.

In this work, GR-SiC ceramics were produced via spark 
plasma sintering (SPS). The resulted GR-SiC ceramics were characterized by field emission scanning electron microscopy (FESEM) and Raman spectroscopy. The dielectric properties including the real permittivity $\left(\varepsilon^{\prime}\right)$, imaginary permittivity $\left(\varepsilon^{\prime \prime}\right)$, ac-conductivity $\left(\sigma_{a c}\right)$ and dielectric loss tangent $(\tan \delta)$ were also studied. The resulted GR-SiC ceramics showed the characteristics of $\mathrm{SiC}$ ceramic and metamaterials, and the synergistic effects of GR and $\mathrm{SiC}$ on negative permittivity of the ceramics were investigated in detail.

\section{Experimental}

\subsection{Chemicals and reagents}

Graphene (purity $98 \%$, thickness $<1 \mathrm{~nm}$, sheet size $20 \times 20$ $\mu \mathrm{m})$ was purchased from Jinan Moxi New Materials Tech. Co., Ltd. Silicon carbide (purity $98 \%$, density $3.22 \mathrm{~g} / \mathrm{cm}^{3}$ ) was purchased from Sinopharm Chemical Reagent Co., Ltd., China.

\subsection{Preparation of GR-SiC ceramics}

The GR and $\mathrm{SiC}$ powders were uniformly mixed by a mechanical ball milling process. After mixing for $2 \mathrm{~h}$, the homogeneously $\mathrm{GR} / \mathrm{SiC}$ mixed powders were obtained, which with different $\mathrm{GR}$ contents were defined as $\mathrm{GR}_{\mathrm{X}} / \mathrm{SiC}(\mathrm{x}=4.33$, $6.30,10.81,13.1,17.27 \mathrm{wt} \%)$. $\mathrm{GR}_{X} / \mathrm{SiC}$ mixed powders were sintered by spark plasma sintering (SPS-211Lx, Japan) with a heating rate of $100{ }^{\circ} \mathrm{C} / \mathrm{min}$ up to $1600{ }^{\circ} \mathrm{C}$ at $50 \mathrm{MPa}$ for $5 \mathrm{~min}$ in vacuum $(<5 \mathrm{~Pa})$ to obtain the $\mathrm{GR}_{\mathrm{X}}-\mathrm{SiC}$ ceramics. Finally, the obtained $\mathrm{GR}_{\mathrm{X}}-\mathrm{SiC}$ ceramics were polished to obtain a sheet sample having a diameter of $20 \mathrm{~mm}$ and a thickness of about $1.5 \mathrm{~mm}$.

\subsection{Characterization}

FESEM (Hitachi SU-70, Tokyo, Japan) was used to observe the microstructure of the $\mathrm{GR}_{\mathrm{X}}-\mathrm{SiC}$ ceramics. Raman spectrometer (InVia, Renishaw, excited by a $532 \mathrm{~nm} \mathrm{He}-\mathrm{Ne}$ laser with a laser spot size of $1 \mu \mathrm{m}^{2}$ ) was used to evaluate the disorder and graphitic symmetry of GR after spark plasma sintering. Dielectric properties, including dielectric constant $\left(\varepsilon^{\prime}\right.$ and $\left.\varepsilon^{\prime \prime}\right)$, dielectric loss tangent $(\tan \delta)$, alternative current conductivity $\left(\sigma_{a c}\right)$ were analyzed by a precision impedance analyzer (Agilent E4991A) with the 16453A dielectric test fixture and the test frequency ranged from $10 \mathrm{MHz}$ to $1 \mathrm{GHz}$. These electrical parameters can be calculated by the corresponding formulas: ${ }^{[4]}$

$$
\begin{gathered}
\varepsilon^{\prime}=\frac{C_{p} t}{\varepsilon_{0} S} \\
\varepsilon^{\prime \prime}=\frac{t}{\omega \varepsilon_{0} S R_{p}} \\
\tan \delta=\frac{\varepsilon^{\prime \prime}}{\left|\varepsilon^{\prime}\right|} \\
\sigma_{a c}=\frac{t}{S R_{p}}
\end{gathered}
$$

where $t$ is the thickness of the sample, $C_{p}$ is the capacitance, $R_{p}$ is the resistance, $\omega(\omega=2 \pi f)$ is the angular frequency, $\varepsilon_{0}$ is the absolute dielectric constant under vacuum $\left(8.85 \times 10^{-12}\right.$ $\mathrm{F} \cdot \mathrm{m}^{-1}$ ), and $S$ is the effective measurement area of sample. The capacitor $C_{p}$, the resistor $R_{p}$ and the reactance $Z^{\prime \prime}$ were directly tested at an alternative current voltage of $100 \mathrm{mV}$.

\section{Results and discussion}

\subsection{Morphologies of the GR-SiC ceramics}

FESEM was used to observe the cross section of GR-SiC ceramics with different GR contents. Fig. 1a shows the internal morphology of the pure $\mathrm{SiC}$ ceramic. Obviously, it was composed of large amounts of sub-wavelength $\mathrm{SiC}$ grains (around $50 \mathrm{~nm}$ ), but the contact between the grains was not tight. Figs. 1 b-f represent the images of $\mathrm{GR}_{4.33}-\mathrm{SiC}, \mathrm{GR}_{6.30}$ $\mathrm{SiC}, \mathrm{GR}_{10.81}$-SiC, $\mathrm{GR}_{13.10}-\mathrm{SiC}$ and $\mathrm{GR}_{17.27}$-SiC, respectively. It could be seen that GR was uniformly dispersed and distributed at grain boundaries of the $\mathrm{SiC}$ matrix. From these images, extraction, bridging and deflection of GR in the $\mathrm{SiC}$ matrix were also observed, and multiple GR sheets entangled into "GR clusters". ${ }^{[10]}$ At the same time, the grain size of $\mathrm{SiC}$ gradually decreased with the GR content increasing, and the porosity of $\mathrm{SiC}$ ceramics further reduced. It was speculated that GR played a role in refining crystal grains, which also promoted the aggregation and fusion of fine $\mathrm{SiC}$ spherulites. ${ }^{[11-}$ ${ }^{14]}$ It was worth noting that, GR overlapped each other into an integral three-dimensional conductive network under high GR content, which will contribute to the improvement of the electrical conductivity of the GR-SiC ceramics.

\subsection{Raman characterization of the GR-SiC ceramics}

The Raman spectra of GR-SiC ceramics with different GR contents are shown in Fig. 2. All samples exhibited two distinguishable peaks assigned to $D$ - and $G$-bands at approximately $1346 \mathrm{~cm}^{-1}$ and $1582 \mathrm{~cm}^{-1}$, respectively, and possessed different $\mathrm{I}_{\mathrm{D}} / \mathrm{I}_{\mathrm{G}}$ values. The $D$-peak represents the defect of the $\mathrm{C}$ atomic lattice, the $G$-peak represents the inplane stretching vibration of $\mathrm{sp}^{2}$ hybridization belonged to $\mathrm{C}$ atom, and the intensity ratio of $D$ and $G$ bands $\left(I_{D} / I_{G}\right)$ was usually used to estimate the disorder and recovery of the $\mathrm{sp}^{2}$ domain of GR. ${ }^{[15-17]} \mathrm{Yu}$ et al. elaborated the relationship between the lateral dimension of "GR clusters" and $I_{D} / I_{G}$ values in detail, and it could be explained according to the Ferrari-Roberson equation: ${ }^{[18]}$

$$
\frac{I_{D}}{I_{G}}=C^{\prime}(\lambda) L_{a}^{2}
$$

where $C^{\prime}(\lambda)=C_{0}+\lambda C_{1}\left(C_{0}=-12.6 \mathrm{~nm}\right.$ and $\left.C_{l}=0.033\right), \lambda$ is the wavelength of the excitation source, $L_{a}$ is the lateral dimension of the "GR" cluster, $I_{D}$ is the intensity of the $D$ band, $I_{G}$ is the intensity of the $G$ band. With the increase of GR content, the $D$-peak width narrowed and peak intensity increased, but the $G$-peak broadened and peak intensity decreased, resulting in the increasing $I_{D} / I_{G}$ values. Therefore, we inferred that the lateral dimension of the "GR" clusters was proportional to GR concentration. In other words, the higher GR concentration, the larger lateral dimension of the "GR" clusters, and this was consistent with the results from FESEM. Through the spark plasma sintering, the original small amounts of oxygen-containing groups on the GR surface was 

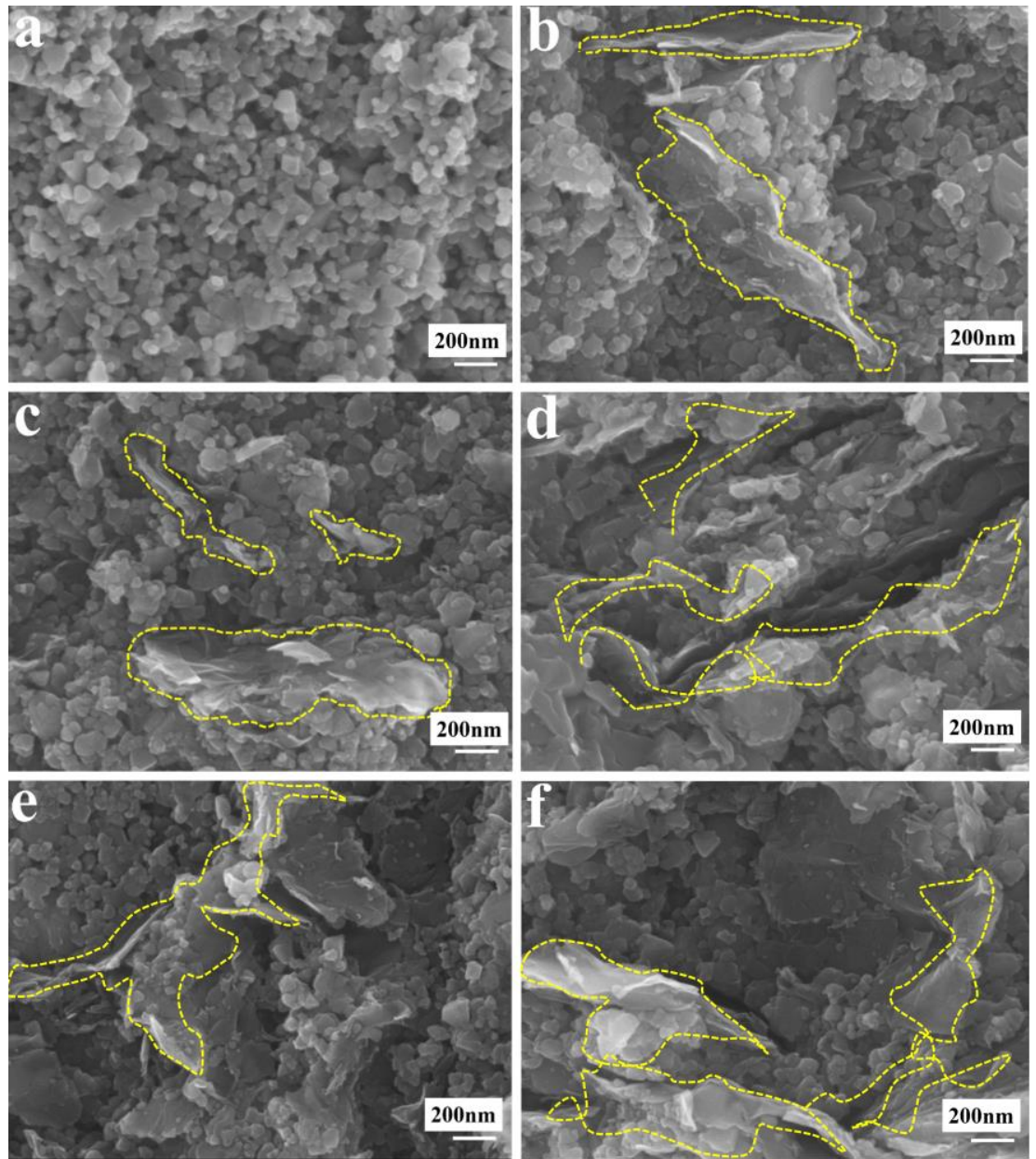

Fig. 1 FESEM images of GR-SiC ceramics with different GR contents: (a) GR ${ }_{0}-\mathrm{SiC}$; (b) GR $\mathrm{GR}_{4.33}-\mathrm{SiC}$; (c) $\mathrm{GR}_{6.30}-\mathrm{SiC}$; (d) $\mathrm{GR}_{10.81}-\mathrm{SiC}$; (e) $\mathrm{GR}_{13.10}$ - $\mathrm{SiC}$; (f) $\mathrm{GR}_{17.27}-\mathrm{SiC}$.

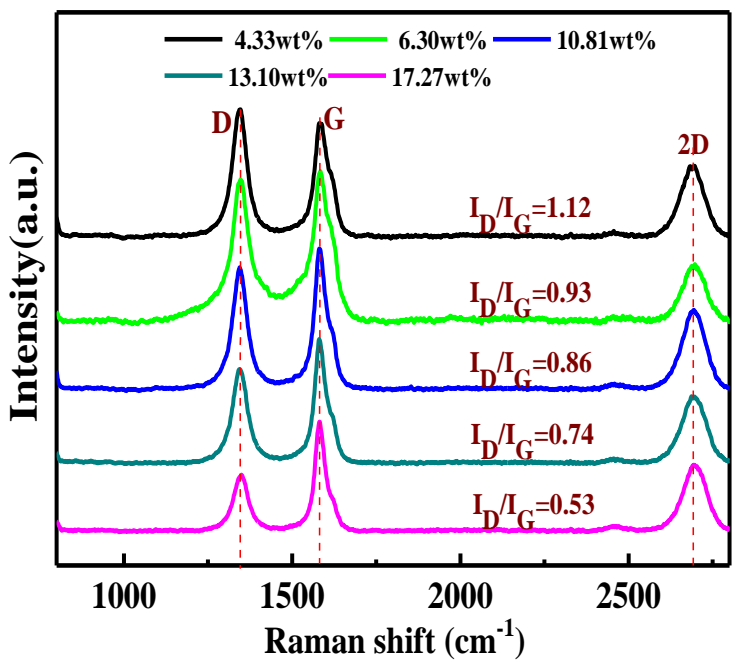

Fig. 2 Raman spectra of GR-SiC ceramics with different GR contents.

removed under high temperature, and the loss of oxygencontaining groups made the ordered carbon atom arrangement disarranged, leading to an increase in defects. ${ }^{[19-21]}$ Interestingly, the "GR" clusters with lager lateral size were more easily connected into a three-dimensional conductive network and successfully integrated into the $\mathrm{SiC}$ ceramic matrix, indicating that the size of the "GR" clusters directly affected conductivity of the GR-SiC ceramics.

\subsection{AC Conductivity and Percolation of GR-SiC ceramics}

Fig. 3a shows the frequency dependence of $\sigma_{a c}$ for GR-SiC ceramics with different GR contents. The $\sigma_{a c}$ of $\mathrm{GR}_{0}-\mathrm{SiC}$, $\mathrm{GR}_{4.33}-\mathrm{SiC}, \mathrm{GR}_{6.30}-\mathrm{SiC}$ was low, because a little amount of GR was isolated from each other in the $\mathrm{SiC}$ matrix. It was difficult to form a conductive path and only possible to be conductive by electronic jump under the action of an electric field, so there is no significant change in the conductivity of the material. The $\sigma_{a c}$ of $\mathrm{GR}_{10.81}-\mathrm{SiC}$ was sharply improved compared to $\mathrm{GR}_{6.30}$-SiC. This sharp change in conductivity was called electrical percolation phenomenon, and the percolation threshold was between $6.30 \mathrm{wt} \%$ and $10.81 \mathrm{wt} \%$ (Fig. 3b). As shown in Fig. 3c, after the GR content was close to the threshold, a large number of GR were in contact with each other to form a complete conductive network, exhibiting a metal-like conductive behavior under the action of an electric field, and the electrical conductivity increased significantly. ${ }^{[19,22]}$ In addition, the $\sigma_{a c}$ of GR-SiC followed the formula (6): ${ }^{[23]}$

$$
\sigma_{a c}=\sigma_{d c}+A \omega^{n}
$$

where $\sigma_{d c}$ is the direct current conductivity, $\omega$ is the angular 

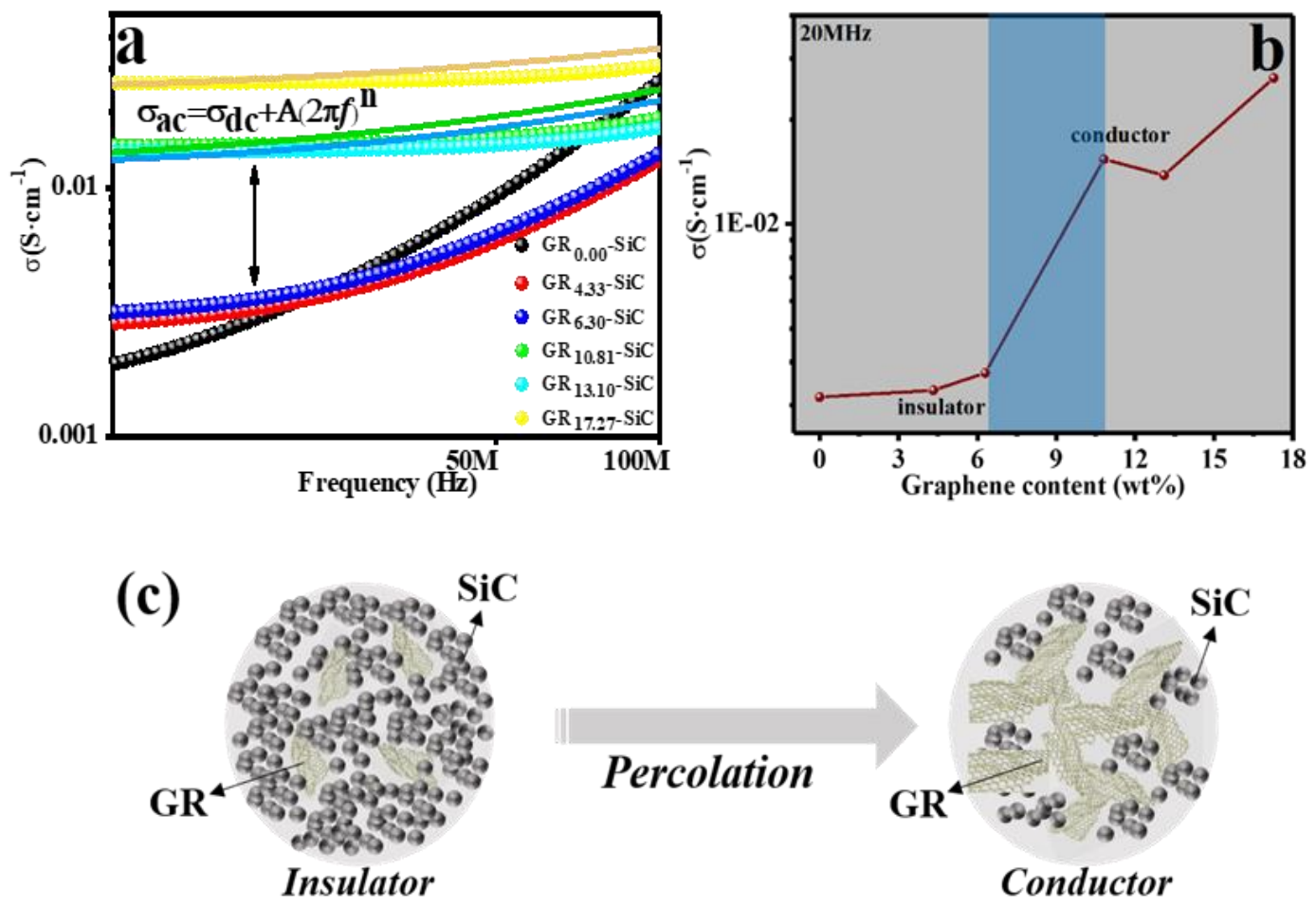

Fig. 3 (a) Frequency dependence of $\sigma_{a c}$ for GR-SiC ceramics with different GR contents; (b) AC conductivity ( $\left.\sigma_{a c}\right)$ for GR-SiC ceramics with different GR contents at $20 \mathrm{MHz}$; (c) Schematic diagram of the structure related to the percolation.

frequency, $n$ is exponent for electrical conductivity and $A$ is the pre-exponential factor. The $\sigma_{a c}-f$ curves were fitted according to formula (6), and it was found that the $\sigma_{a c} f$ curves of $\mathrm{GR}_{10.81}-\mathrm{SiC}, \mathrm{GR}_{13.10}-\mathrm{SiC}$ and $\mathrm{GR}_{17.27}-\mathrm{SiC}$ were in great agreement with the fitting curves and the reliability factor $R^{2}$ were $0.94,0.92$ and 0.90 , respectively.

\subsection{Dielectric properties of the GR-SiC ceramics}

Fig. 4a shows the frequency dependence of $\varepsilon\left(\varepsilon^{\prime}\right.$ and $\left.\varepsilon^{\prime \prime}\right)$ for GR-SiC composite ceramics with different GR contents. The $\varepsilon^{\prime}-f$ curve of the pure $\mathrm{SiC}$ ceramic showed a strong discretestate resonance dielectric resonance in the high-frequency band, ${ }^{[24]}$ which may be related to the magnetic dipole mode and scattering characteristics of sub-wavelength $\mathrm{SiC}$ grains. ${ }^{[25-}$ ${ }^{28]}$ The $\varepsilon^{\prime}-f$ curves of GR-SiC ceramics also displayed a Fanolike resonance phenomenon, which may be caused by interference between the continuous-state resonance mode of GR and the discrete-state resonance mode of SiC. ${ }^{[29,30]}$ The mathematical model of Fano-like scattering resonance is shown in formula (7):

$$
\sigma \approx \frac{\left(q \Gamma_{r e s} / 2+E-E_{r e s}\right)^{2}}{\left(\Gamma_{r e s} / 2\right)^{2}+\left(E-E_{r e s}\right)^{2}}
$$

where $\sigma$ represents the total cross-sectional area of the scattering, $\Gamma_{\text {res }}$ is the peak width of the resonant energy, and $q$ is the Fano variable. From Fig. 4a, it was found that the amplitude of resonance gradually decreased with the increase of GR content, which may be due to the coherent coupling between the surface plasmon of GR and the excitation response of $\mathrm{SiC}$ semiconductor. Meanwhile, the mutual dielectric screening between $\mathrm{SiC}$ and GR greatly inhibited the plasmon resonance of GR. ${ }^{[24,31]}$ When the continuous-state resonance mode of GR was completely suppressed until it disappeared, $q$ became infinite, and the Fano scattering resonance model will become a Lorentz scattering resonance model as shown in formula (8): $:^{[32]}$

$$
\begin{gathered}
\sigma \approx \frac{\left(q \Gamma_{r e s} / 2\right)^{2}}{\left(\Gamma_{r e s} / 2\right)^{2}+\left(E-E_{r e s}\right)^{2}} \\
\varepsilon^{\prime}=1+\frac{\omega_{p}^{2}\left(\omega_{0}^{2}-\omega^{2}\right)}{\left(\omega_{0}^{2}-\omega^{2}\right)^{2}+\omega^{2} \Gamma_{L}^{2}}
\end{gathered}
$$

where $\omega_{p}\left(\omega_{p}=2 \pi f_{p}\right)$ is the plasma oscillation angular frequency describing the resonance intensity, $f_{p}$ is the plasma oscillation frequency, $\omega_{0}\left(\omega_{0}=2 \pi f_{0}\right)$ is the resonance angular frequency, $f_{0}$ is the resonance frequency, $\omega(\omega=2 \pi f)$ is the angular frequency of the applied electromagnetic field, and $\Gamma_{L}$ is the damping constant associated with the resonance. Lorentz dielectric resonance model was very similar to Lorentz scattering resonance model as shown in formula (9). The $\varepsilon^{\prime}-f$ curves were fitted according to formula (9), we found that the $\varepsilon^{\prime}-f$ curve of $\mathrm{GR}_{17.27} \mathrm{SiC}$ (the mass fraction of GR was 17.27 $\mathrm{wt} \%$ ) was in good agreement with the fitting curve and the reliability factor $R^{2}$ were 0.9 . This indicated that the Fano resonance model changed to the Lorentz dielectric resonance model. In addition, positive and negative dielectric transitions caused by dielectric resonance were also observed in the real dielectric curve. The transition frequencies of $\mathrm{GR}_{4.33}-\mathrm{SiC}$, $\mathrm{GR}_{6.30}-\mathrm{SiC}, \mathrm{GR}_{10.81}-\mathrm{SiC}, \mathrm{GR}_{13.10}-\mathrm{SiC}$, and $\mathrm{GR}_{17.27} \mathrm{SiC}$ were 486, 462, 332, 351 and $206 \mathrm{MHz}$, respectively. It was worth noting that the dielectric transition frequency gradually shifted to the lower frequency with the content of GR increasing, indicating that the negative dielectric bandwidth extended 

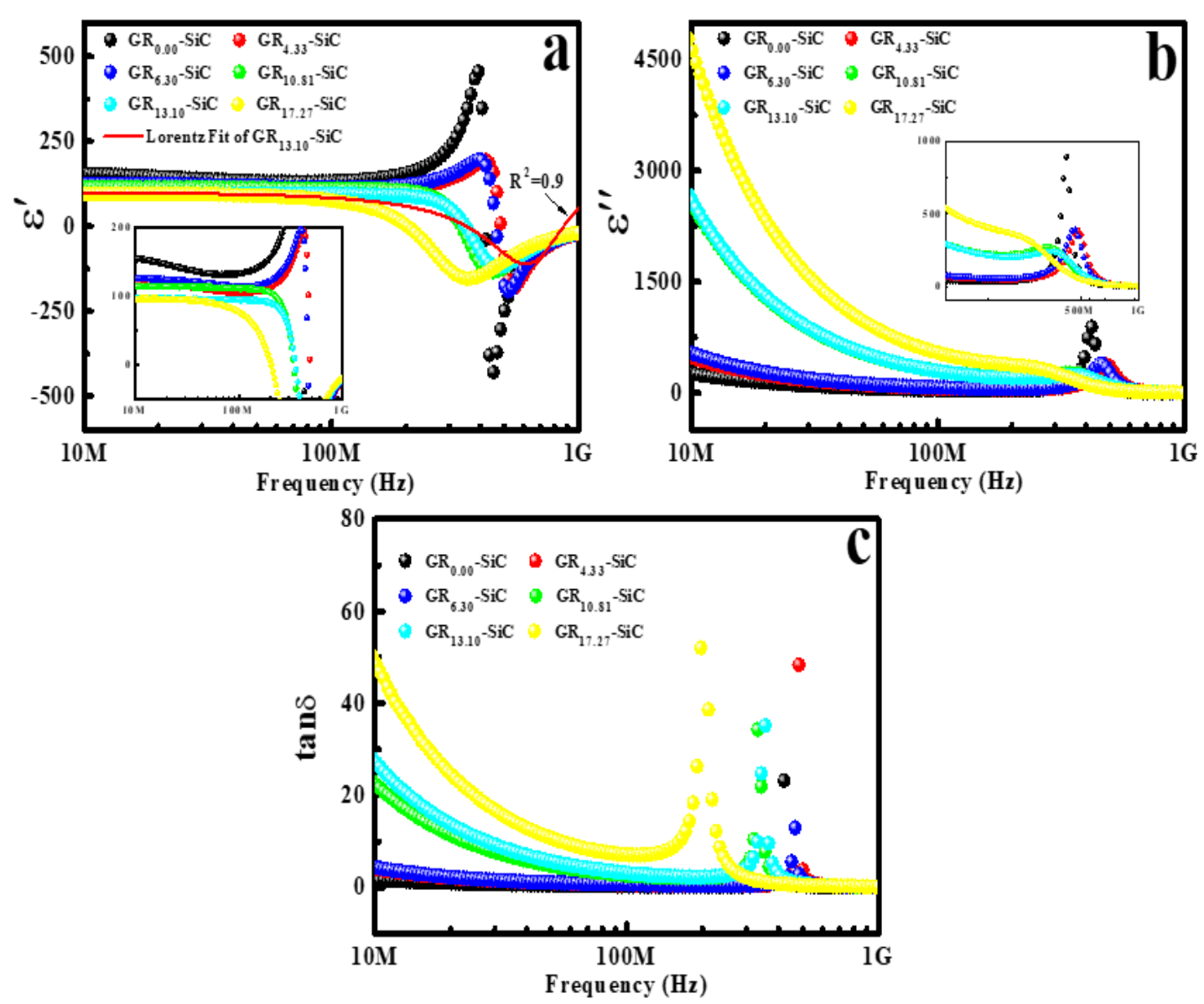

Fig. 4 (a) Frequency dependence of $\varepsilon^{\prime}$ for GR-SiC ceramics with different GR contents; (b) Frequency dependence of $\varepsilon^{\prime \prime}$ for GR-SiC ceramics with different GR contents; (c) Frequency dependence of $\tan \delta$ for GR-SiC ceramics with different GR contents.

wider. Moreover, the bandwidth was also controlled by the GR contents in the GR-SiC ceramics.

Fig. $4 \mathrm{~b}$ shows the relationship between $\varepsilon^{\prime \prime}$ and $f$. The $\varepsilon^{\prime \prime}$ was known as the dissipation factor, which reflected the dielectric loss of the material. Usually, the dielectric loss was mainly affected by the conductive process, dipole and interfacial polarization in the radio-frequency band. ${ }^{[33-35]}$ Meanwhile, the interfacial polarization disappeared under the action of high frequency external electric field below $1 \mathrm{MHz}$. Therefore, we only considered the conduction loss and dipole loss. The $\varepsilon^{\prime \prime}$ could refer to the following formula: ${ }^{22]}$

$$
\begin{gathered}
\varepsilon^{\prime \prime}=\varepsilon_{c}{ }^{\prime \prime}+\varepsilon_{p}{ }^{\prime \prime} \\
\varepsilon_{c}{ }^{\prime \prime}=\frac{\sigma_{d c}}{\varepsilon_{0} \omega} \\
\varepsilon_{p}{ }^{\prime \prime}=\frac{\varepsilon_{s}-\varepsilon_{\infty}}{1+\omega^{2} \tau^{2}} \omega \tau
\end{gathered}
$$

where $\varepsilon_{c}$ " represents the conduction loss, $\varepsilon_{p}$ " represents the dipole loss, $\sigma_{d c}$ is the direct current conductivity, $\varepsilon_{s}$ is the staticfrequency permittivity, $\tau$ was the relaxation time. Pure $\mathrm{SiC}$ ceramics had low dielectric loss, while the $\varepsilon^{\prime \prime}$ of GR-SiC composites increased significantly with the enhancement of GR content, which was attributed to the $\varepsilon_{c}{ }^{\prime \prime}$. The GR conductive network was gradually improved with the continuous increase of GR content in the $\mathrm{SiC}$ matrix. Therefore, the electrons accumulated at the $\mathrm{GR} / \mathrm{SiC}$ interface were gradually weakened by the influence of the Coulomb blocking effect, and are electromagnetically induced to generate eddy currents under the action of an electric field, resulting in greater conduction loss. ${ }^{[36-38]}$ This indicated that $\varepsilon_{c}{ }^{\prime \prime}$ played a major role in dielectric loss of GR-SiC ceramics. The $\tan \delta\left(\tan \delta=\varepsilon^{\prime \prime} / \varepsilon^{\prime}\right)$ represented the ratio of energy loss to energy storing, Fig. $4 \mathrm{c}$ shows the relationship between $\tan \delta$ and $f$. The $\tan \delta$ of the GR-SiC ceramics with contents of $4.33,6.30,10.81$, 13.10 , and $17.27 \mathrm{wt} \%$ showed extremely large peaks around 486, 462, 332, 351 and $206 \mathrm{MHz}$, respectively, which were consistent with the transition frequencies of the $\varepsilon^{\prime}$ from positive to negative. Moreover, dielectric resonance caused the appearance of energy loss peaks for $\tan \delta-f$ curves. ${ }^{[32]}$

\section{Conclusions}

GR-SiC ceramics with tunable broadband of negative permittivity were fabricated by spark plasma sintering process. On one hand, GR interconnected into "GR clusters" with the content increasing, and uniformly dispersed in the $\mathrm{SiC}$ matrix due to sufficient ball milling. On the other hand, $\mathrm{SiC}$ was gradually refined by the suppression of GR at the grain boundary, which further increased the density of the GR-SiC composite ceramic. Besides, Raman results showed that the increase in the lateral dimension of the "GR" cluster was proportional to the GR concentration. When the GR content 
exceeded $6.30 \mathrm{wt} \%$, a significant electrical percolation phenomenon occurred, and the conductive mechanism changed to a free electron model. Fano-like resonance, generated by the mutual interference between the continuousstate resonance mode of GR and the discrete-state resonance mode of $\mathrm{SiC}$, exhilaratingly transformed into the Lorentz model with GR content increasing. In addition, owing to the mutual dielectric screening greatly between $\mathrm{SiC}$ and GR, the dielectric transition frequency gradually shifted to the lower frequency with the content of GR increasing, and the negative dielectric band became wider. Prospectively, it will greatly expand the effective bandwidth of metacomposites used for strong coupling, electromagnetic wave absorption, beam antenna, etc.

\section{Conflict of interest}

There are no conflicts to declare.

\section{Supporting information}

Not applicable.

\section{References}

[1] T. Ergin, N. Stenger, P. Brenner, J. -B. Pendry, M. Wegener, Science, 2010, 328, 337-339, doi: 10.1126/science.1186351.

[2] B. Zhao, C. Park, J. Mater. Chem. C, 2017, 5, 6954-6961, doi: $10.1039 / \mathrm{c} 7 \mathrm{tc} 01865 \mathrm{~g}$.

[3] A. Moreau, C. Ciracì, J. Mock, R. Hill, Q. Wang, B. Wiley, A. Chilkoti, D. Smith, Nature, 2012, 492, 86-89, doi: 10.1038/nature11615.

[4] R. Yin, X. Huang, L. Qian, Ceram. Int., 2019, 45, 56535659, doi: 10.1016/j.ceramint.2018.12.030.

[5] C. Cheng, R. Fan, G. Fan, H. Liu, J. Zhang, J. Shen, Q. Ma, R. Wei, Z. Guo, J. Mater. Chem. C, 2019, 7, 3160-3167, doi: $10.1039 / \mathrm{c} 9 \mathrm{tc} 00291 \mathrm{j}$

[6] K. Järrendahl, R. Davis, Semiconduct. Semimet., 1998, 52, 1-20, doi: 10.1016/S0080-8784(08)62843-4.

[7] P. -P. Yu, Z. -J. Lin, J. Yu, J. Eur. Ceram Soc., 2021, 41, 6859-6868, doi: 10.1016/j.jeurceramsoc.2021.07.020.

[8] T. Xiao, J. -L. Kuang, Q. -F. Zheng, S. -X. Xiong, W. -X. Liu, W. -B. Cao, J. Alloy. Compd., 2020, 831, 154753, doi: 10.1016/j.jallcom.2020.154753.

[9] Z. Wang, K. Sun, P. Xie, Y. Liu, R. -H. Fan, J. PhysCondens. Mat., 2017, 29, 365703, doi: 10.1088/1361648X/aa7b5d.

[10] J. Liu, H. -X. Yan, J. -R Mike, K. Jiang, J. Eur. Ceram Soc., 2012, 32, 4185-4193, doi: 10.1016/j.jeurceramsoc.2012.07.007.

[11] K. Wang, Y. -F. Wang, T. Wei, Mater. Res. Bull., 2011, 46, 315-318, doi: 10.1016/j.materresbull.2010.11.005.

[12] C. Ramirez, P. Miranzo, M. Belmonte, M. Osendi, P. Poza, S. Vega-Diaz, M. Terrones, J. Eur. Ceram. Soc., 2014, 34, 161169, doi: 10.1016/j.jeurceramsoc.2013.08.039.

[13] O. Tapaszto, L. Tapaszto, M. Marko, F. Kern, R. Gadow, C. Balazsi, Chem. Phys. Let., 2011, 511, 340-343, doi: 10.1016/j.cplett.2011.06.047.
[14] Q. -S. Li, Y. -J. Zhang, H. -Y. Gong, H. -B. Sun, T. Li, X. Guo, S. -H. Ai, Ceram. Int., 2015, 41, 13547-13552, doi: 10.1016/j.ceramint.2015.07.149.

[15] Y. Wang, Y. Du, R. Qiang, C. Tian, P. Xu, X. Han, $A d v$. Mater. Interfaces, 2016, 3, 1500684, doi: 10.1002/admi.201500684

[16] V. Zolyomi, J. Koltai, J. Kurti, Phys. Status Solidi B, 2011, 248, 2435-2444, doi: 10.1002/pssb.201100295.

[17] X. Zheng, Y. Peng, Y. Yang, J. Chen, H. Tian, X. Cui, W. Zheng, J. Raman Spectrosc., 2017, 48, 97-103, doi: 10.1002/jrs.4998.

[18] Y. Yu, F. Xia, Q. Huang, J. Fang, L. An, J. Am. Ceram. Soc., 2017, 100, 5113- 5119, doi: 10.1111/jace.15025.

[19] X. Huang, R. Yin, L. Qian, W. Zhao, Ceram. Int., 2019, 45, 17784-17792, doi: 10.1016/j.ceramint.2019.05.349.

[20] G. Pierin, C. Grotta, P. Colombo, C. Mattevi, J. Eur. Ceram. Soc., 2016, 36, 1589-1594, doi: 10.1016/j.jeurceramsoc.2016.01.047.

[21] X. -F. Wang, M. Gabriela, K. Morita, E. Ionescu, $J$. Ceram. Soc. Jpn., 2016, 124, 981-988, doi: 10.2109/jcersj2.16089.

[22] H. -K. Wu, Y. -Y. Qi, Z. -Y. Wang, W. Zhao, X. -M. Liu, L. Qian, Compos. Sci. Tech., 2017, 151, 79-84, doi: 10.1016/j.compscitech.2017.08.011.

[23] X. Wang, Z. Shi, M. Chen, R. Fan, K. Yan, K. Sun, S. Pan, M. Yu, J. Am. Ceram. Soc., 2014, 97, 3223-3229, doi: 10.1111/jace.13113.

[24] B. Luk'yanchuk, N. -I. Zheludev, S. -A. Maier, N. -J. Halas, P. Nordlander, H. Giessen, C. -T. Chong, Nature Mater., 2010, 9, 707-715, doi: 10.1038/NMAT2810.

[25] K. -Q. Le, A. Alù, J. Bai, J. Appl. Phys., 2015, 117, 023118, doi: 10.1063/1.4905619.

[26] Y. -P. Zhang, T. -T. Li, B. -B. Zeng, H. -Y. Zhang, H. -H. Lv, X. -Y. Huang, W. -L. Zhang, A. -K. Azad, Nanoscale, 2015, 7, 12682-12688, doi: 10.1039/c5nr03044g.

[27] C. Zhao, L. Cui, X. Song, Y. Li, J. Xiao, Opt. Commun., 2015, 340, 1-4, doi: 10.1016/j.optcom.2014.11.081.

[28] A. -E. Miroshnichenko, Y. -S. Kivshar, Nano Lett., 2012, 12, 6459-6463, doi: 10.1021/n1303927q.

[29] S. -D. Liu, Z. Yang, R. -P. Liu, X. -Y. Li, ACS Nano, 2012, 6, 6260-6271, doi: 10.1021/nn3017052.

[30] J. -A. Fan, C. Wu, K. Bao, J. -M. Bao, R. Bardhan, N. -J. Halas, V. -N. Manoharan, P. Nordlander, G. Shvets, F. Capasso, Science, 2010, 328, 1135-1135, doi: 10.1126/science.1187949. [31] C. Yi, T. Kim, W. Jiao, Y. Yang, A. Lazarides, K. Hingerl, G. Bruno, A. Brown, M. Losurdo, Small, 2012, 8, 2721-2730, doi: 10.1002/smll.201200694.

[32] C. Cheng, R. Fan, Y. Ren, T. Ding, L. Qian, J. Guo, X. Li, L. An, H. Lei, Y. Yin, Z. Guo, Nanoscale, 2017, 9, 5779-5787, doi: $10.1039 / \mathrm{c} 7 \mathrm{nr} 01516 \mathrm{j}$.

[33] M. Cao, X. Wang, W. Cao, X. Fang, B. Wen, J. Yuan, Small, 2018, 14, 1800987, doi: 10.1002/smll.201800987.

[34] W. Cao, X. Wang, J. Yuan, W. Wang, M. Cao, J. Mater. Chem. C, 2015, 3, 10017-10022, doi: 10.1039/c5tc02185e.

[35] M. Cao, Y. Cai, P. He, J. Shu, W. Cao, J. Yuan, Chem. Eng. 
J., 2019, 359, 1265-1302, doi: 10.1016/j.cej.2018.11.051.

[36] G. -H. Fan, Z. -Y. Wang, H. Ren, Y. Liu, R. -H. Fan, Scripta Mater., 2021, 190, 1-6, doi: 10.1016/j.scriptamat.2020.08.027.

[37] G. -H. Fan, Z. -Y. Wang, K. Sun, Y. Liu, R. -H. Fan, J. Mater. Sci. Technol., 2021, 61, 125-131, doi: 10.1016/j.jmst.2020.06.013.

[38] G. -H. Fan, Z. -Y. Wang, G. -D. Zhang, Y. Liu, R. -H. Fan, Compos. Commun., 2021, 24, 100667, doi: 10.1016/j.coco.2021.100667.

Publisher's Note: Engineered Science Publisher remains neutral with regard to jurisdictional claims in published maps and institutional affiliations. 